\title{
Risk management of power portfolios and valuation of flexibility
}

\section{Journal Article}

\section{Author(s):}

Doege, Jörg; Schiltknecht, Philippe; Lüthi, Hans-Jakob

Publication date:

2006-04

Permanent link:

https://doi.org/10.3929/ethz-b-000002573

Rights / license:

In Copyright - Non-Commercial Use Permitted

Originally published in:

OR Spectrum 28(2), https://doi.org/10.1007/s00291-005-0005-4 


\title{
Risk management of power portfolios and valuation of flexibility
}

Published online: 6 January 2006

(C) Springer-Verlag 2006

\begin{abstract}
Risk management by applying operational flexibility is becoming a key issue for production companies. This paper discusses how a power portfolio can be hedged through its own production assets. In particular we model operational flexibility of a hydro pump storage plant and show how to dispatch it to hedge against adverse movements in the portfolio. Moreover, we present how volume risk, which is not hedgeable with standard contracts from power exchanges, can be managed by an intelligent dispatch policy. Despite the incompleteness of the market we quantify the value of this operational flexibility in the framework of coherent risk measures.
\end{abstract}

Keywords Risk management - Coherent risk measures · Dispatch management of power plants · Operational flexibility

\section{Introduction}

The electricity market in Europe is going through a big transition. From being a regulated market with no or very low uncertainty in future earnings the market is now becoming liberalized and deregulated. Electricity prices are no longer determined by the regulator but by market participants. From deregulated markets (e.g., California) one has observed extremely volatile prices (see Borenstein and Bushnell 1999). For a power generation company this fact makes future profits very uncertain and evocates a strong need for risk management.

Electricity contracts can be traded at power exchanges - similarly to stocks e.g., in Germany. There are, however, major differences between traditional fi-

This research project is gratefully supported by the Swiss Innovation Promotion Agency KTI/CTI, Berne $(\mathrm{CH})$.

J. Doege $\cdot$ P. Schiltknecht $\cdot$ H.-J. Lüthi $(\bowtie)$

Institute for Operations Research, Department of Mathematics, ETH Zürich,

Clausiusstrasse 45, 8092 Zürich, Switzerland

E-mail: luethi@ifor.math.ethz.ch 
nancial markets and the electricity market such as non-storability of electricity and physical transmission constraints implying market incompleteness (see Güssow 2001). Due to the specific characteristics of electricity such as price jumps and the fact that power generation companies are by nature long, risk management ideas developed for financial markets are not directly applicable to the electricity market. In this paper, we will try to shed some light on one part of power risk management, namely the interaction of physical production and financial contracts.

A typical power producer is exposed to volume risk mainly stemming from the system load of a supply area and bilateral agreements. Given the fact that only futures and plain options, which cannot fully hedge volume risk, are traded at power exchanges, a utility can use its production capacity as a hedging tool to offset both volume and price risk. Thereby the hedging performance and consequently the income from production strongly depends on the flexibility of its generation facility.

In the last few years a wide range of literature dealing with electricity risk management was published. Essentially, this research can be divided into two main parts: pricing and valuation. Pricing is a macroeconomic approach that is dealing with determining the fair market price of contracts by assuming risk neutrality. Thus, major literature on pricing (see Eydeland and Geman 2000; Deng et al. 2001; or Clewlow and Strickland 2001) mainly deals with future or forward contracts and plain options written on futures (see Hinz et al. 2004). The spot price being the underlying asset was neglected so far. This is due to the fact that spot markets, which imply immediate physical delivery, are incomplete and hence no unique risk-neutral probability measure can be found. Additionally, the convenience yield and the cost and carry relation which are needed for commodities in order to assume no-arbitrage cannot be applied due to the non-storability of electricity (see Geman 2001).

Latest pricing approaches from Jaillet et al. (2004) or Carmona and Dayanik (2004) deal with swing options, which are an extension of take-or-pay options (see Thompson 1995). Swing options are nowadays heavily traded at OTC markets with the spot price being the underlying. This fact makes pricing for these kinds of options extremely difficult. Only Carmona and Dayanik (2004) have developed a closed-form solution for various spot price models so far. Kamat and Oren (2002) focused on a slightly different type of options, namely exotic options on interruptible contracts. Recently there have also been approaches of portfolio optimization in a macroeconomic framework making use of equilibrium models (see Hinz 2003).

Of crucial importance for pricing methodologies on options with the spot price being the underlying is a risk-neutral spot price model. Those models can only be estimated by incorporating futures contracts. The first mean-reverting models for the objective ${ }^{1}$ probability measure were introduced by Pilipovic (1997) and expanded by Eydeland and Geman (2000). Schwartz and Lucia (2002) were able to estimate a mean-reverting stochastic spot price model for both probability measures. Kholodnyi (2004) included in his model price jumps. Latest approaches incorporate a stochastic system load factor in order to obtain price jumps (see Burger et al. 2004) and can be estimated for both probability measures. This methodology had first been mentioned by Eydeland and Geman (2000).

\footnotetext{
${ }^{1}$ i.e. non-risk-neutral.
} 
Valuation is the micro-economic equivalent for pricing and deals with determining the value of a portfolio from a producer's point of view. This means that all generation facilities and the corresponding connectivity have to be modeled individually and in detail. In contrast to pricing methods all computations in valuation approaches are done by using the objective probability measure assuming that spot prices are externally given by scenarios. Latest approaches used (multi-stage) stochastic programming (see Fleten et al. 2002; Güssow 2001) wherein a more strategic point of view was taken by having e.g., an annual decision basis. GröweKuska and Römisch (2002) developed a model to focus on the short-run, i.e. hourly decision basis. In stochastic programming decisions are path-dependent, i.e. they are made given the realization of the random variable for this period. The advantage is that the strategy on how to operate the utility can be adjusted over time which results in a dynamic dispatch strategy. This unavoidably leads to a size problem, i.e. that the number of scenarios being used in the short-run optimization (i.e. hourly decisions) is very small (e.g., in Gröwe-Kuska and Römisch 2002 only nine scenarios were used). Moreover, stochastic programming approaches are concentrated on expected values without looking at risk.

The approach we are suggesting is different from stochastic programming and an extension of Unger (2002) who first developed this method by incorporating coherent risk measures in a linear portfolio optimization framework where operational as well as contractual decisions are considered. There are basically two advantages of this methodology: First, the expected profit is maximized given that the overall risk will never exceed a certain level. Secondly, the computational ease of certain coherent risk measures and linear programming which allows for a large number of scenarios and fast computation time.

Having set up a linear portfolio optimization model in Section 2, we apply it to derive a dispatch policy for the most flexible power plant, namely the hydro pump storage. Furthermore, we will introduce the novel notion of flexibility by exploiting the dual problem in Section 3. Our main contribution is to present a hands-on methodology to value a hydro pump storage plant's flexibility based on the work of Lüthi and Doege (2005). We will give a guideline on how to examine this specific value of flexibility and on how to use it for daily operations and management decisions. It explicitly shows the flexibility in terms of risk reduction capability of a given system. Moreover investment decisions concerning the enhancement of flexibility can be easily valued and evaluated ${ }^{2}$ with this powerful technique. The article is concluded in Section 4.

\section{Power portfolio optimization}

In the electrical power industry a power portfolio can be divided into two parts: namely the contract portfolio consisting of spot, futures, and OTC-contracts on electricity, and the production portfolio which is represented by the generation facilities (c.f. Fig. 1). While the optimization of a contract portfolio is well-known from financial theory (see e.g., Eydeland and Geman 2000) problems arise when

\footnotetext{
${ }^{2}$ Evaluated in this context means to decide on the technical investment or its zero-coupon equivalent. Additionally the optimal level of operations can be determined.
} 


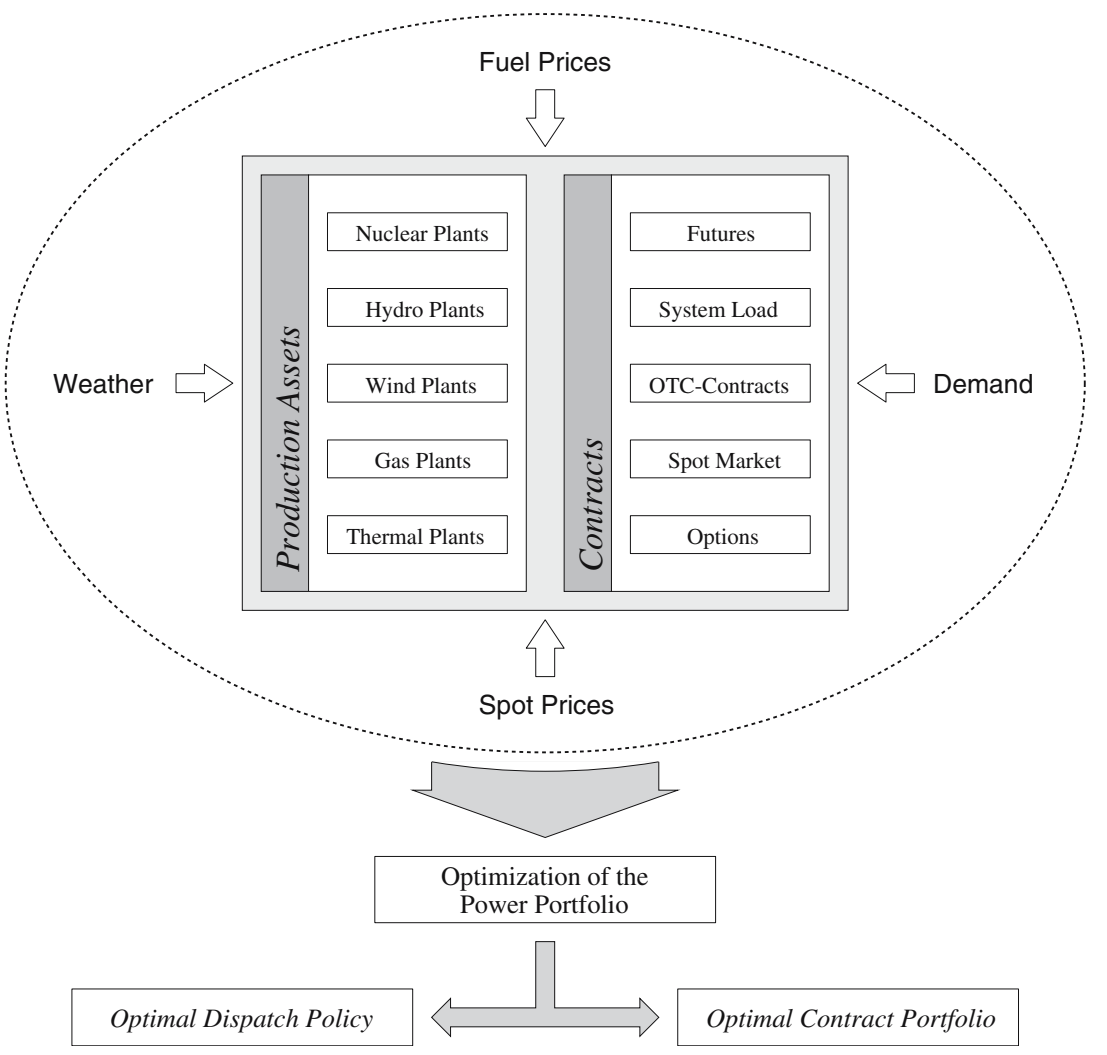

Fig. 1 Schematic figure of portfolio optimization

the whole portfolio is tackled. In the following subsections we will derive an optimization framework that allows us to integrate both portfolios.

A typical production portfolio of a utility consists of baseload generators and peakers (see Stoft 2002). Baseload generators run most of the time and are stopped rarely, whereas peakers have a technology that allows immediate ramp-up. Thus, the main task of the production portfolio is to determine how much capacity should be generated using each type of technology. In the following, we assume that the production portfolio of the utility company consists of a nuclear power plant representing baseload generators and a hydro pump storage representing peakers. Regarding the contract portfolio we suppose that the utility has access to a futures and spot market and is assumed to have a local supply area.

In the next subsection we will first focus on the particularities of hydro plants and show how information-responsive operating strategies (so-called dispatch policies) can be determined in order take advantage of volume and price volatility which cannot be covered by the nuclear power plant. In Subsection 2.2 we will then focus on risk management aspects while in Subsection 2.3 we will merge operational decisions from Subsection 2.2 and additional contractual considerations into a optimization framework. 


\subsection{Hydro storage plant and dispatch policy}

The management of a hydro pump storage as production asset is an interesting and complex problem. While the inflexible nuclear plant covers a certain baseload the hydro pump storage can be used for peakload and excess power at high price periods. Its flexibility stems from the fact that on the one hand water is a storable commodity whereas electricity is not, and on the other hand from its ability to change output immediately ${ }^{3}$ at nearly no marginal costs. The hydro management involves a continuous decision process whether to release water (i.e. producing electricity) now or to store it and release it later. If pumps are installed the owner, additionally, has the possibility (option) to pump water.

In the following, let $T$ be the time period in which we want to study the exercise strategy of a hydro pump storage plant. Since day-ahead electricity is traded on a discrete $^{4}$ time axis we work in a discrete world and the time horizon is divided into $T$ periods $t=0, \ldots, T$.

As already mentioned, in each period the owner of a hydro pump storage has the option to produce at marginal costs. Intuitively, one would like to produce in every point in time (see Mas-Colell et al. 1995). By exercising the option to produce, the water level decreases, and as water inflow is stochastic, the probability to produce in the future decreases, whereas pumping effects the opposite. The decision about producing or pumping will thus affect future possibilities of dispatching the plant. Figure 2 tries to visualize this whereby $x_{\max }^{+}$denotes the maximum capacity of the turbines and $x_{\max }^{-}$the maximum capacity of the pumps respectively.

It is straightforward, that the way how the hydro pump storage plant is dispatched strongly affects profit and loss. Accordingly, the question arises what is a good way of dispatching the hydro plant and how to determine the right moments for production or pumping. According to the Fig. 1 it is plausible that the dispatch strategy should depend on the external drivers spot price $S_{t}$, demand $D_{t}$, and aggregated water inflow $I_{t}^{a}=\sum_{i=1}^{t} I_{i}$.

Unfortunately it is not evident how an appropriate dispatch strategy should look like. As shown in Rudin (1976) any real valued function can be approximated by a linear combination of step functions and hence any dispatch function $x^{+}$and $x^{-}$ can be approximated accordingly. Choosing step functions $g_{i}^{+}$and $g_{i}^{-}, i=1, \ldots, m$, and weighting factors $\gamma^{+}=\left(\gamma_{1}^{+}, \ldots, \gamma_{\mathrm{m}}^{+}\right), \gamma^{-}=\left(\gamma_{1}^{-}, \ldots, \gamma_{\mathrm{m}}^{-}\right)$satisfying $\gamma^{+}, \gamma^{-} \geq 0$ and $\sum_{i=1}^{m} \gamma_{i}^{+}=\sum_{i=1}^{m} \gamma_{i}^{-}=1, x_{t}^{+}$and $x_{t}^{-}$can then be written as follows:

$$
x_{t}^{+} \approx \sum_{i=1}^{m} \gamma_{i}^{+} g_{i}^{+} \text {and } x_{t}^{-} \approx \sum_{i=1}^{m} \gamma_{i}^{+} g_{i}^{-} \text {. }
$$

As stated above, these exercise functions should solely be determined by (stochastic) external factors. As the dynamics of the dispatch strategy is induced by these random variables the step functions have to be defined accordingly. This can

\footnotetext{
${ }^{3}$ Ramp-up times are within $10 \mathrm{~s}$.

${ }^{4}$ Spot prices are determined for each hour of the next day. This is done on hourly auctions.
} 


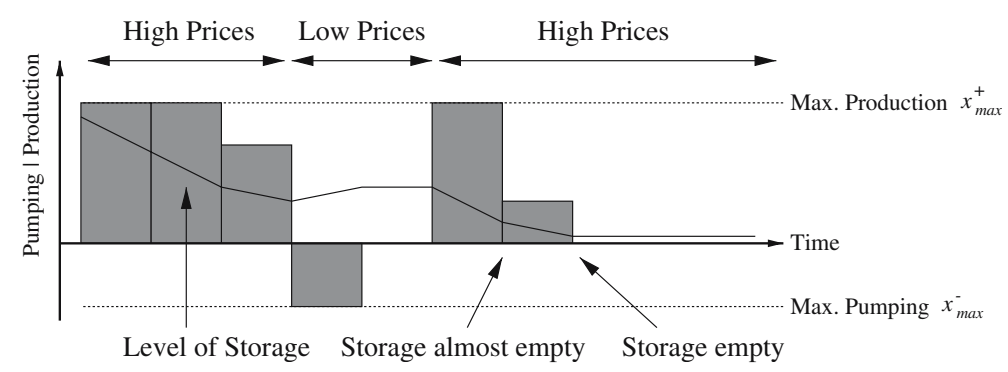

Fig. 2 Illustration of the dispatch of a hydro storage plant

be achieved by defining $g_{i}^{+}$and $g_{i}^{-}$as follows by introducing two families of predetermined thresholds tuples $\left(S_{t, i}^{+}, D_{t, i}^{+}, I_{t, i}^{a+}\right)_{i=1, \ldots, m}$ and $\left(S_{t, i}^{-}, D_{t, i}^{-}, I_{t, i}{ }^{a-}\right)_{i=1, \ldots, m}$ respectively for every period $t$

$$
\begin{aligned}
& g_{i}^{+}\left(S_{t}, D_{t}, I_{t}^{a}\right)= \begin{cases}x_{\max }^{+} & S_{t} \geq S_{t, i}^{+} \text {and } D_{t} \geq D_{t, i}^{+} \text {and } I_{t}^{a} \geq I_{t, i}^{a+}, \\
0 & \text { otherwise }\end{cases} \\
& g_{i}^{-}\left(S_{t}, D_{t}, I_{t}^{a}\right)= \begin{cases}0 & S_{t} \geq S_{t, i}^{-} \text {or } D_{t} \geq D_{t, i}^{-} \text {or } I_{t}^{a} \geq I_{t, i}^{a-}, \\
x_{\max }^{-} & \text {otherwise }\end{cases}
\end{aligned}
$$

Applying Eq. (1), the exercise functions can then be approximated as:

$$
x_{t}^{+} \approx \sum_{i=1}^{m} \gamma_{i}^{+} g_{i}^{+}\left(S_{t}, D_{t}, I_{t}^{a}\right) \text { and }=x_{t}^{-} \approx \sum_{i=1}^{m} \gamma_{i}^{-} g_{i}^{-}\left(S_{t}, D_{t}, I_{t}^{a}\right)
$$

The quality of the approximation strongly depends on the number $m$ of thresholds as well as on the their values. While theoretically a huge number of thresholdfamilies would be preferable, with respect to the practical implementation, upcoming in Subsection 2.3, it is more beneficial to keep the number of thresholds small in order to guarantee its feasibility and reduce computation time.

Regarding the optimization framework presented in Subsection 2.3 the key of this dynamic approach is to let the weighting factors $\gamma=\left(\gamma^{+}, \gamma^{-}\right)$be the decision variables which implicitly determine the amount of water used for production and pumping respectively. The optimization problem yields the best convex combination of the-exogenously given-exercise functions $g_{i}^{+}$and $g_{i}^{-}$.

Note, that given a time horizon of e.g., one year electricity prices, demand, and inflow have seasonal pattern (e.g., in Europe prices and demand are generally higher in winter compared to summer times). Such seasonality could be taken into account by introducing seasonal weighting factors $\gamma_{k}=\left(\gamma_{k}^{+}, \gamma_{k}^{-}\right)$with $k$ being the season and dividing the time period $1, \ldots, T$ into $K$ disjoint "seasons" $\tau_{1, \ldots,} \tau_{\mathrm{k}}$, i.e.

$$
\bigcup_{k=1}^{K} \tau_{k}=\{1, \ldots, T\}, \tau_{k_{1}} \cap \tau_{k_{2}}=\emptyset \quad \forall k_{1} \neq k_{2}
$$


For every season $k$, we admit a different, i.e. we have

$$
\begin{array}{ll}
\gamma_{k}^{+}=\left(\gamma_{1, k}^{+}, \ldots \gamma_{m, k}^{+}\right) & k=1, \ldots, K \\
\gamma_{k}^{-}=\left(\gamma_{1, k}^{-}, \ldots \gamma_{m, k}^{-}\right) & k=1, \ldots, K
\end{array}
$$

Therefore, Eq. (1) becomes

$$
\begin{array}{ll}
x_{t}^{+}:=\sum_{i=1}^{m} \sum_{k=1}^{K} \gamma_{i, k}^{+} 1_{\left\{t \in \tau_{k}\right\}} g_{i, t}^{+}\left(S_{t}, D_{t}, I_{t}^{a}\right) & \forall t \\
x_{t}^{-}:=\sum_{i=1}^{m} \sum_{k=1}^{K} \gamma_{i, k}^{+} 1_{\left\{t \in \tau_{k}\right\}} g_{i, t}^{-}\left(S_{t}, D_{t}, I_{t}^{a}\right) & \forall t
\end{array}
$$

It follows immediately for every $k$, that

$$
\sum_{i=1}^{m} \gamma_{i, k}^{+}=1 \quad \forall k, \quad \sum_{i=1}^{m} \gamma_{i, k}^{-}=1 \quad \forall k .
$$

\subsection{Risk measures}

In the traditional financial markets the standard portfolio optimization approach is the mean-variance portfolio problem as described by Markowitz (1952) where the portfolio variance is minimized subject to a constraint on the expected return. Variance has a well-documented motivation as a risk measure and is reasonable to be the natural risk measure resulting from expected utility maximization (see Arrow 1971). It would hence be tempting to use the mean-variance approach also in the electricity market. Eydeland and Wolyniec (2002) and Eberlein and Stahl (2003) show that a power portfolio, however, differs substantially from a traditional financial portfolio, due to non-normal returns, the involvement of production assets, non-storability of electricity, and complex contracts. This is why a nonsymmetric risk measure has to be used instead of variance. Value-at-Risk (VaR), the most famous example for those kind of risk measures, is not an appropriate choice due to the lack of subadditivity ${ }^{5}$ which has been proven by Delbaen (2000). ${ }^{6}$ Hence a good non-symmetric risk measure should have properties like coherence (see Artzner et al. 1999) or convexity (see Föllmer and Schied 2002). Thus, for computational ease Conditional Value-at-Risk (CVaR), first introduced by Rockafellar and Uryasev (2000), is an appropriate measure of risk that fulfills the coherence properties.

In the following, a short overview of the concept of CVaR will be given. During this conceptual discussion we mainly refer to the continuous case. Later on we will, based on the work of Rockafellar and Uryasev (2000), show how the CVaR approach is inearized and applied in a LP framework.

\footnotetext{
${ }^{5}$ Subadditivity is probably the most important property to be a good risk measure for portfolios. ${ }^{6}$ These results have been numerically shown by Acerbi and Tasche (2002) and Frey and McNeil (2002).
} 
Let the random variable $l(\mathbf{x}, \mathbf{Y})$ denote the loss function of a decision variable $\mathbf{x} \in \mathbb{R}^{n}$, which can be seen as a portfolio, and a given random vector $\mathbf{Y} \in \mathbb{R}^{m}$ representing the future values of stochastic variables such as e.g., spot price with distribution $p(\mathbf{Y})$. Let $\operatorname{VaR}_{\beta}$ denote the $\beta$-quantile of the induced loss \& profit distribution. The risk measure $\mathrm{CVaR}$ with confidence level $\beta$ is defined by the conditional expectation

$$
\begin{aligned}
\mathrm{CVaR}_{\beta}(\mathbf{x}) & :=\mathbb{E}\left[l(\mathbf{x}, \mathbf{Y}) \mid l(\mathbf{x}, \mathbf{Y}) \geq \operatorname{VaR}_{\beta}\right] \\
& =(1-\beta)^{-1} \int_{l(\mathbf{x}, \mathbf{Y}) \geq \operatorname{VaR}_{\beta}(\mathbf{x})} l(\mathbf{x}, \mathbf{Y}) p(\mathbf{Y}) d \mathbf{Y}
\end{aligned}
$$

which, as we assume, is well defined for any choice $\mathbf{x} \in \mathbf{X}^{7}$ (cf. Fig. 3).

Given the set of all feasible portfolios $\mathbf{X} \subseteq \mathbb{R}^{n}$ and assuming that we want to maximize the expected profit while controlling our risk (measured with CVaR with a confidence level of $\beta$ ), the optimization problem can be written as follows:

$$
\begin{array}{ll}
\max & \mathbb{E}[-l(\mathbf{x}, \mathbf{Y})] \\
\text { s.t. } & \mathrm{CVaR}_{\beta}(\mathbf{x}) \leq C \\
& \mathbf{x} \in \mathbf{X}
\end{array}
$$

At first view this problems seems to be difficult to solve. However, Rockafellar and Uryasev (2000) and Bertsimas et al. (2000) have shown that under the assumption that $\mathbf{Y}$ does not depend on $\mathbf{x}^{8}, l(\mathbf{x}, \mathbf{Y})$ is linear (convex) in $\mathbf{x}$ and the feasible set $\mathbf{X}$ is a polyhedral (convex) set, the problem (6) can be solved by linear (convex) programming. In particular Rockafellar and Uryasev (2000) show that $\mathrm{CVaR}$ as defined in Eq. (5) can be characterized by the following convex optimization problem $\mathrm{CVaR}_{\beta}(\mathbf{x})=\min _{\alpha \in \mathbb{R}} F_{\beta}(\mathbf{x}, \alpha)$, where $F_{\beta}(\mathbf{x}, \alpha)$ is given by

$$
F_{\beta}(\mathbf{x}, \alpha):=\alpha+(1-\beta)^{-1} \int_{\mathbf{Y} \in \mathbb{R}^{n}}[l(\mathbf{x}, \mathbf{Y})-\alpha]^{+} p(\mathbf{Y}) d \mathbf{Y}
$$

If we generate $J$ scenarios, $j=1, \ldots, J$ with realizations $\omega_{1}, \ldots, \omega_{J}$ of the random variable $\mathbf{Y}^{9}, F_{\beta}(\mathbf{x}, \alpha)$ can be approximated by a convex piecewise linear function in $\alpha$ :

$$
\tilde{F}_{\beta}(\mathbf{x}, \alpha)=\alpha+\frac{1}{J(1-\beta)} \sum_{j=i}^{J}\left[l\left(\mathbf{x}, \omega_{\mathbf{j}}\right)-\alpha\right]^{+} .
$$

\footnotetext{
${ }^{7}$ See Rockafellar and Uryasev (2002) for the mathematical assumptions.

8 i.e. we assume that the strategy of a single power generation company will not influence spot markets.

${ }^{9}$ In our case for every scenario $j, \omega_{\mathrm{j}}$ corresponds to a joint path of the stochastic values, spot price $S_{t}$, demand $D_{t}$ and inflow $I_{t}$, over all periods $t=1, \ldots, T$.
} 


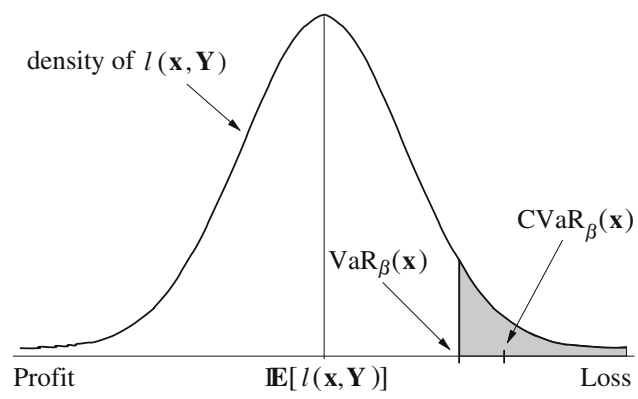

Fig. 3 Graphical interpretation of CVaR for given $\mathbf{x} \in \mathbf{X}$

Using the auxiliary variables $z_{1}, \ldots, z_{J} \in \mathbb{R}$ the optimization problem (6) can be approximated by

$$
\begin{gathered}
\max -\frac{1}{J} \sum_{j=1}^{J} l\left(\mathbf{x}, \omega_{\mathbf{j}}\right) \\
\text { s.t. } \alpha+\frac{1}{(1-\beta) J} \sum_{j=1}^{J} z_{j} \leq C \\
z_{j} \geq l\left(\mathbf{x}, \omega_{\mathbf{j}}\right)-\alpha, j=1, \ldots, J \\
\alpha \in \mathbb{R}, \mathbf{x} \in \mathbf{X}, z_{j} \geq 0, j=1, \ldots, J .
\end{gathered}
$$

Assuming linearity of $l$ in $\mathbf{x}$, the initial problem, as stated in problem (6), becomes an instance of linear programming. ${ }^{10}$ We will use this powerful framework in the following as the main tool to derive optimal dispatch policies. Needless to state that modeling the stochastics of risk driving processes such as spot price, demand (for both see Burger et al. 2004), and inflow is of crucial importance but will not be treated in detail in this paper.

\subsection{Optimization model}

According to our conceptual consideration presented in previous subsections, we can now set up our model. The first step of the optimization model is to describe the production portfolio as outlined in Section 2. We assume that the produced electricity of the nuclear power plant $B L_{t}$ at time $t$ is known in advance. This is due to the fact that this kind of baseload power plants have a must-run condition and they can only be shut down for maintenance reasons. With respect to hydro pump storages the particularities have already been examined and discussed in Section 2.1.

The contract portfolio of the utility is on the one hand represented by spot and futures contracts, where $S_{t, j}$ [Euro/MWh] denotes the spot price in period $t$ given scenario $j$. There exist $n$ different futures contracts in the market. The position in each of them is denoted by $x_{1}^{F}, \ldots, x_{n}^{F}[\mathrm{MWh} /$ period] respectively. The utility may go long or short in any of these traded futures contracts. Since different futures

\footnotetext{
${ }^{10}$ A more rigorous mathematical analysis is presented in Rockafellar and Uryasev (2000).
} 
have distinct periods of underlying base or peak-load contracts we denote by $X_{t}^{F}$ the set of futures which has a base or peakload contract in period $t$ as underlying.

Another important part of the contract portfolio is the supply area where the utility company has a fixed price delivery commitment. Let the stochastic demand, which is often called system load, in period $t$ and scenario $j$ be denoted by $D_{t, j}$ [MWh]. Because of the Kirchhoff laws the utility has to be in balance in the sense that the amount of electricity that is bought or produced has to be equal to the electricity that is sold or used in each period of time $t$ :

$$
D_{t, j}=\underbrace{\underbrace{B L_{t}}_{\text {Base }}+\underbrace{x_{t, j}^{+}}_{\text {Prod. Hydro }}-\underbrace{x_{t, j}^{-}}_{\text {Pump. Hydro }}}_{\text {Production Portfolio }}+\underbrace{\sum_{\text {Futures }}^{\sum_{i}^{F} \in X_{t}^{F}} x_{i}^{F}-\underbrace{x_{t, j}^{\text {spot }}}_{\text {Spot-Market }}}_{\text {Fontract Portfolio }}
$$

Therefore the spot position $x_{t, j}^{\text {spot }}$, which is uniquely determined by the dispatch of the hydro pump storage plant, the produced electricity of the nuclear plant, the futures positions, and the local supply area demand, is defined as

$$
x_{t, j}^{\text {spot }}:=B L_{t}-x_{t, j}^{+}+\frac{x_{t, j}^{-}}{\chi}-\sum_{x_{i}^{F} \in X_{t}^{F}} x_{i}^{F}+D_{t, j}
$$

where $\chi$ denotes the efficiency of a pump, defined as the ratio between pumped energy in terms of water and used electrical energy.

In order to set up the loss function one has to specify the corresponding marginal costs according to Section 2:

Marginal production costs Denote the associated marginal production costs ${ }^{11}$ of a baseload power plant as $c^{B L}$ [Euro/MWh] and its corresponding costs for the hydro pump storage as $c^{+}$[Euro/MWh] for turbining and $c^{-}$[Euro/MWh] for pumping.

Marginal contractual costs Let $c^{D}[$ Euro/MWh] denote the costs associated with the supply area. Those costs are mainly transmission costs and fees. Moreover, we assume that no costs are associated with spot or futures market trading.

Grouping all components depending on the spot price in $x_{t, j}^{\text {spot }}$ as in Eq. (11) and summing up the discounted losses in each period, the total profit $\&$ loss in scenario $j$ is given by

$$
\begin{aligned}
l\left(\mathbf{x}, \gamma, \omega_{j}\right):= & \sum_{t=1}^{T} e^{-t r}(\underbrace{c^{B L} B L_{t}+c^{+} x_{t, j}^{+}+c^{-} x_{t, j}^{-}}_{\text {Production Portfolio }}+ \\
& \underbrace{\sum_{i=1}^{n} F_{i} x_{i}^{F} 1_{x_{i}^{\mathrm{F}} \in X_{t}^{F}+c^{D} D_{t, j}-S_{t, j} x_{t, j}^{s p o t}}}_{\text {Contract Portfolio }}),
\end{aligned}
$$

where $r$ is a one-periodic continuously compounded discount rate.

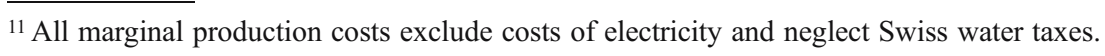


While most components such as objective function contractual decisions, dispatch policy, and risk considerations have already been discussed, the only parts that need some more explanation are the technical constraints of the hydro pump storage. In the following we define $\bar{V}_{T}$ as the average end level of water of the hydro pump storage, $V_{\text {end }}$ as the end level of water that has to be achieved after one year which is typically equal to the beginning water level $V_{0}$ in order to guarantee sustainability, and $V_{\max }$ as the maximum water level, i.e. the dam height. Given the inflow $I_{t, j}$ for every period $t$ and every scenario $j$, the stored energy (i.e. water) $V_{t, j}$, as well as the spill-over $L_{t, j}$, the optimization problem as stated in Eq. (9) can be written in the following manner

$$
\begin{aligned}
& \max -\frac{1}{J} \sum_{j=1}^{J} l\left(\mathrm{x}, \gamma, \omega_{j}\right) \\
& \text { s.t } \quad \alpha+\frac{1}{(1-\beta) J} \sum_{j=1}^{J} z_{j} \leq C \\
& z_{j} \geq l\left(\mathrm{x}, \gamma, \omega_{j}\right)+\alpha \geq 0 \quad \forall j \\
& 0 \leq V_{0}+\sum_{i=1}^{t}\left(I_{i, j}-L_{i, j}-x_{i, j}^{+}+x_{i, j}^{-}\right)=V_{t, j} \quad \forall t, j \\
& V_{\max } \geq V_{0}+\sum_{i=1}^{t}\left(I_{i, j}-L_{i, j}-x_{i, j}^{+}+x_{i, j}^{-}\right)=V_{t, j} \quad \forall t, j \\
& V_{\text {end }} \leq \frac{1}{J} \sum_{j=1}^{J}\left(V_{0}+\sum_{i=1}^{T}\left(I_{i, j}-L_{i, j}-x_{i, j}^{+}+x_{i, j}^{-}\right)\right)=\bar{V}_{T} \\
& V_{0}=V_{\text {end }} \\
& x_{t, j}^{+}=\sum_{i=1}^{m} \gamma_{i}^{+} g_{i}^{+}\left(S_{t, j}, D_{t, j}, I_{t, j}^{a}\right) \quad \forall t, j \\
& x_{t, j}^{-}=\sum_{i=1}^{m} \gamma_{i}^{-} g_{i}^{-}\left(S_{t, j}, D_{t, j}, I_{t, j}^{a}\right) \quad \forall t, j \\
& \sum_{i=1}^{m} \gamma_{i}^{+}=\sum_{i=1}^{m} \gamma_{i}^{-}=1 \quad \forall t, j \\
& \boldsymbol{\gamma}^{+}, \boldsymbol{\gamma}^{-}, L_{t, j} \geq 0 \quad \forall t, j \\
& x_{t}^{F} \in X_{t}^{F} \quad \forall t
\end{aligned}
$$

\subsection{Case study}

Based on a real-world case study, the above optimization model was used to determine the optimal dispatch policy for different risk levels $C$. The time horizon for the optimization model was one year, with the European Energy Exchange (EEX) being the underlying spot and futures market. We did only include 


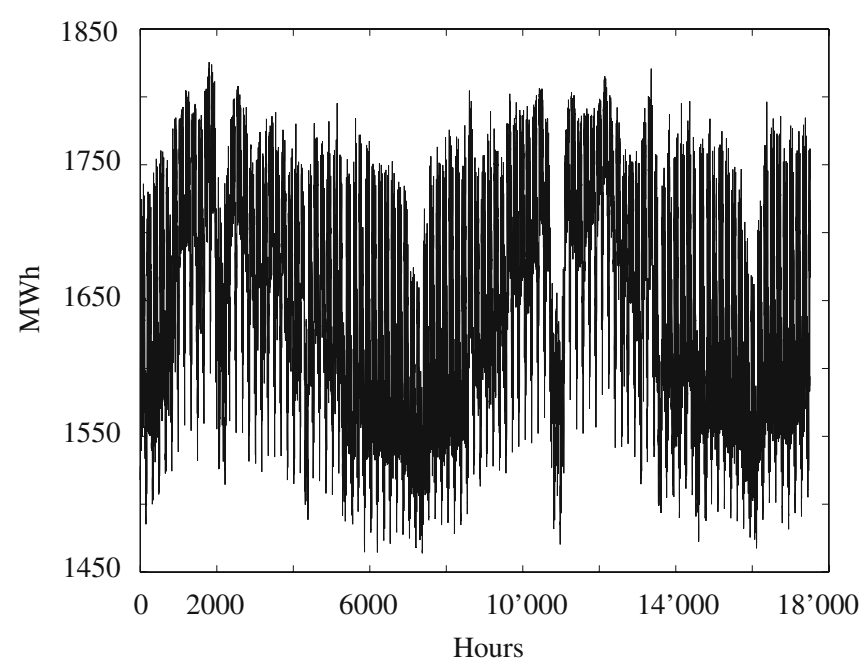

Fig. 4 Demand of a Swiss supply area

monthly ${ }^{12}$ futures contracts from the EEX assuming the futures price to be the average price of the corresponding spot contracts. There is no restriction on the maximum amount of electricity in the futures contracts. Moreover, we assume that the transmission capacities between Germany and Switzerland are large enough at any point in time $t$. Thus, there are no restrictions or congestions. One could easily incorporate transmission constraints but then an additional term called imbalance electricity or reserve power, has to be added in order fulfill the Kirchhoff law. Additionally we took the system load of a Swiss balance zone as demand (see Fig. 4). The stochastics for the input variables demand and spot price where modeled and simulated ${ }^{13}$ according to Burger et al. (2004) on an hourly basis, i.e. $T=8,760$. The inflow followed a stochastic mean-reverting log-normal process.

The nuclear power plant $B L_{t}$ has a given dispatch plan, including maintenance times, with an average production of 1,600 MW per hour. This dispatch plan is known in advance. The maximum capacity of the turbines is $x_{\max }^{+}=225 \mathrm{MW}$ and the capacity of the pumps $x_{\max }^{-}=25 \mathrm{MW}$. The maximum volume of the hydro pump storage is $V_{\max }=35,000 \mathrm{MW}$ and both the beginning as well as the end water level are $80 \%$ of this, hence $V_{0}=V_{\text {end }}=0.8 V_{\text {max }}$.

Figures 6 and 7 show the optimal dispatch, plotted as a function of the spot price and the demand: Figure 6 refers to the optimal dispatch for a risk constraint of 2 million Euro, corresponding to a pure profit maximization. One observes that production in this case is solely a function of the spot price. This leads to the natural hypothesis that for loose risk constraints ${ }^{14}$ the policy is focused only on high profit. A large amount of the water is dispatched during high price periods, while pumping is conducted for low prices and low demand. Figure 7 illustrates

\footnotetext{
${ }^{12}$ Monthly futures contracts have the highest liquidity in the EEX market.

${ }^{13}$ Using Monte-Carlo simulation techniques.

${ }^{14}$ The risk constraint is no longer a binding restriction.
} 


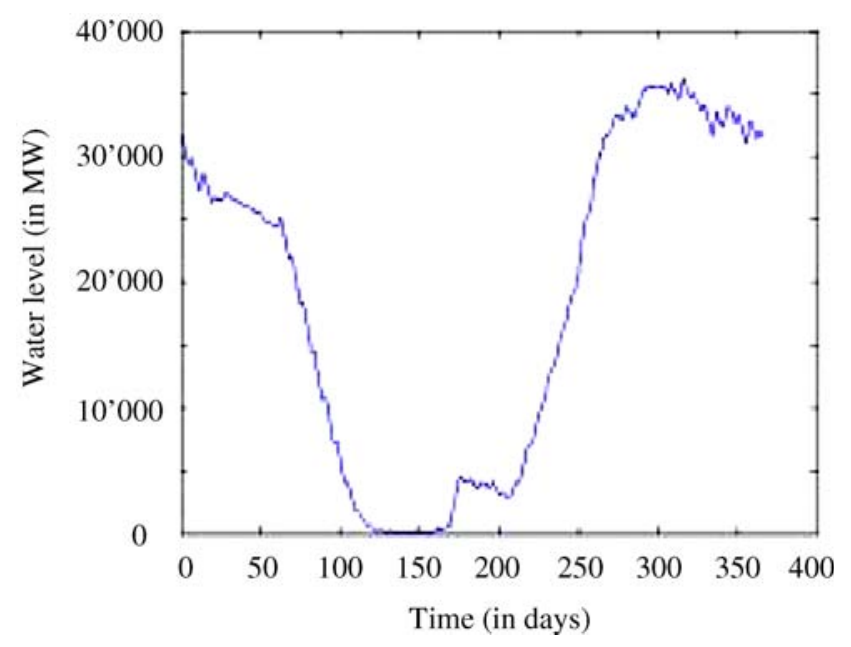

Fig. 5 Average water level for hydro pump storage plant

well that the policy becomes sensitive in dealing with the volume risk when strengthening the risk constraint to -5.6 million Euro.

It is important to note that this dispatch policy is optimal over all scenarios but not necessarily for a single one. This means that if one would compute a policy for each individual scenario than there might be a better policy for the according

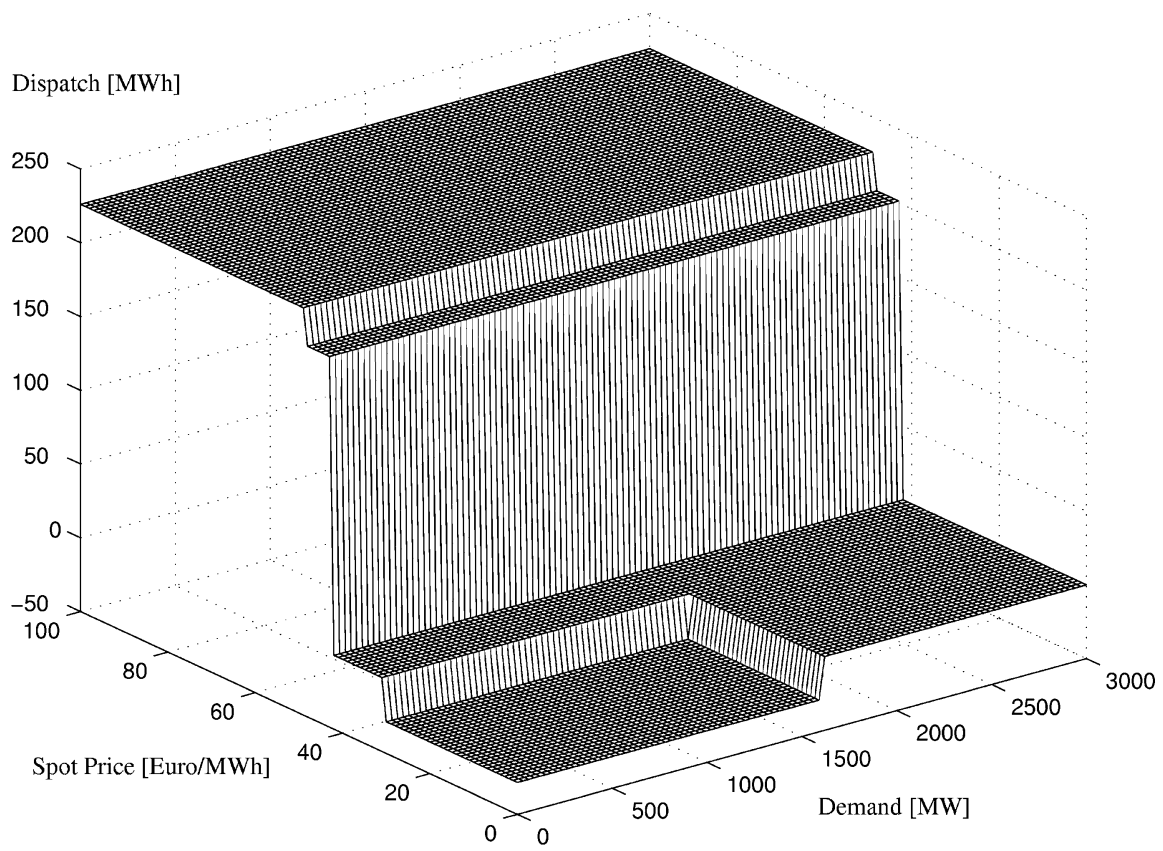

Fig. 6 Optimal dispatch for a risk constraint of 2 million Euro 


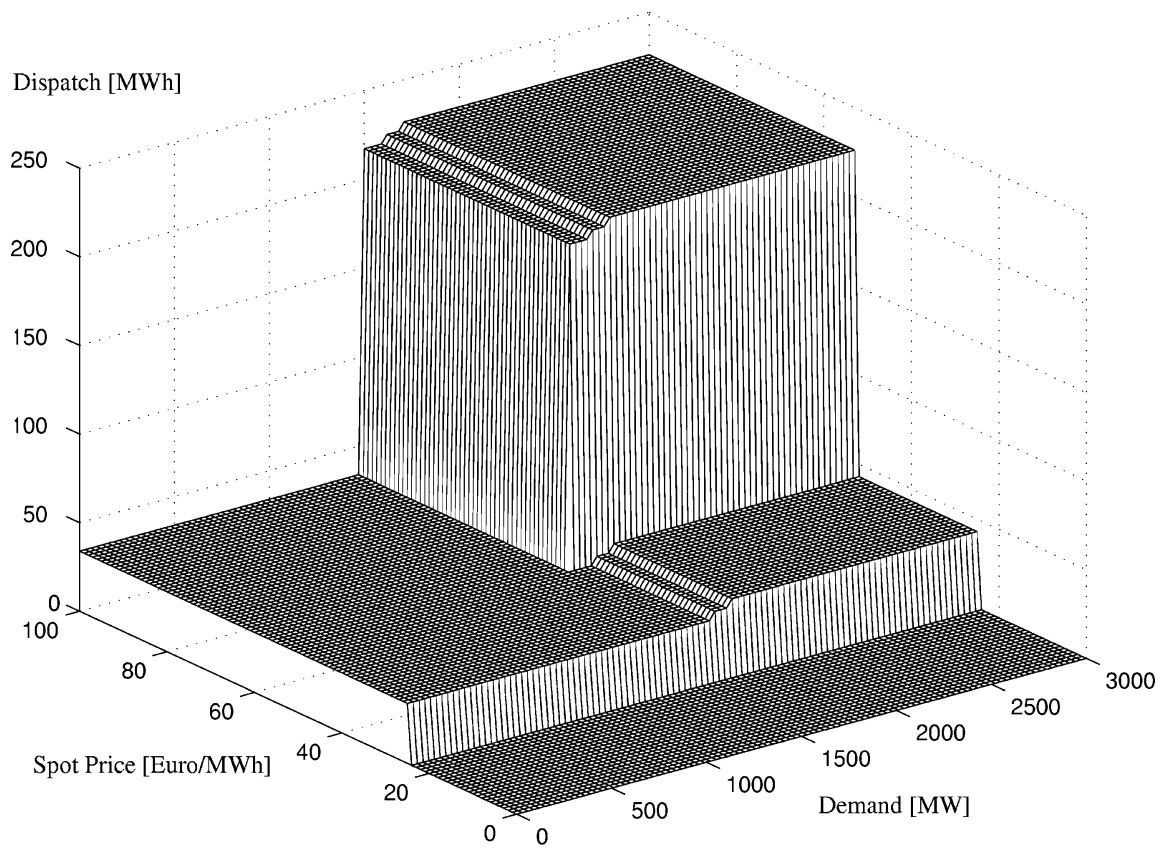

Fig. 7 Optimal exercise functions for a risk constraint of -5.6 million Euro

scenario than the one we obtained from our model. The dispatch policy from our model is the method of choice for operations as it encompasses the entirety of all scenarios that can occur. Applying the dispatch policy from Fig. 6 one immediately obtains the average water level for the hydrological year as shown in Fig. 5. The water level follows a "tub" curve - a typical observation for hydro pump storages.

The size of the optimization problem heavily depends on the number of scenarios being used. In our real-world example we chose $J=1,000$ scenarios and $m=6$ thresholds. There is no rule on choosing the number of thresholds. Theoretically, it is preferably to choose a large number of thresholds which results in much longer computation time. Thus, the choice is a tradeoff between computation time and a slightly higher expected profit. From our experience, $m=6$ has been shown to bea good choice. The linear portfolio optimization portfolio for $m=6, J=1,000$, and $T=8,760$ was implemented and solved with GAMS/CPLEX 9.0. The running time on a compute server with $1.7 \mathrm{GHz}$ and $4 \mathrm{~GB}$ of RAM was approximately $20 \mathrm{~min}$.

\section{Valuation of flexibility}

Flexibility can be defined as the ability to easily respond to unforeseen changes in a variety of ways (see Ku 2003). Before deregulation of electricity markets there was no need to measure "flexibility" as there was nearly no (financial) uncertainty and 
therefore no need to adjust to unexpected changes. Liberalization of markets opened a wide range of uncertainty, and suddenly the financial implication of flexibility became an issue.

In this section we want to focus on the adaptiveness and flexibility of a hydro pump storage, and more precisely on the value associated with this flexibility. From the perspective of a utility there are basically two ways to look at flexibility: First, valuating flexibility by comparing the profit $\&$ loss and risk profile of a hydro pump storage to other technologies with different degrees of flexibility (such as e.g., nuclear power plants), or secondly, and that is the approach used in this paper, by focusing on the intrinsic value of the flexibility and point out the marginal values of different flexibility parameters as a core topic.

By using the sensitivity analysis in the following we focus especially on two flexibility parameters covering the operational as well the strategic level of production management. More precisely, in Subsection 3.1 we will investigate the marginal value of risk (denoted by the according risk level $C$ ) and motivate the socalled hedging value of flexibility, while in Subsection 3.2 we will discuss the marginal value of flexibility (in terms of energy).

\subsection{Hedging value of flexibility}

In Section 2, we derived an optimization model, which simultaneously incorporates three essential aspects of modern electricity production management: flexibility, uncertainty, and risk. Flexibility is taken into account by designing a dynamic dispatch policy (see Eq. 4). The uncertainty is introduced by different underlying scenarios and, with respect to risk, the so-called $\mathrm{CVaR}$ concept is used. By solving the optimization model we obtain an efficient frontier (see Fig. 8) where point A is an optimal portfolio with a risk of -2 million Euro and an expected profit of approximately 7.6 million Euro. The corresponding profit \& loss distribution is given in Fig. 9 on the left.

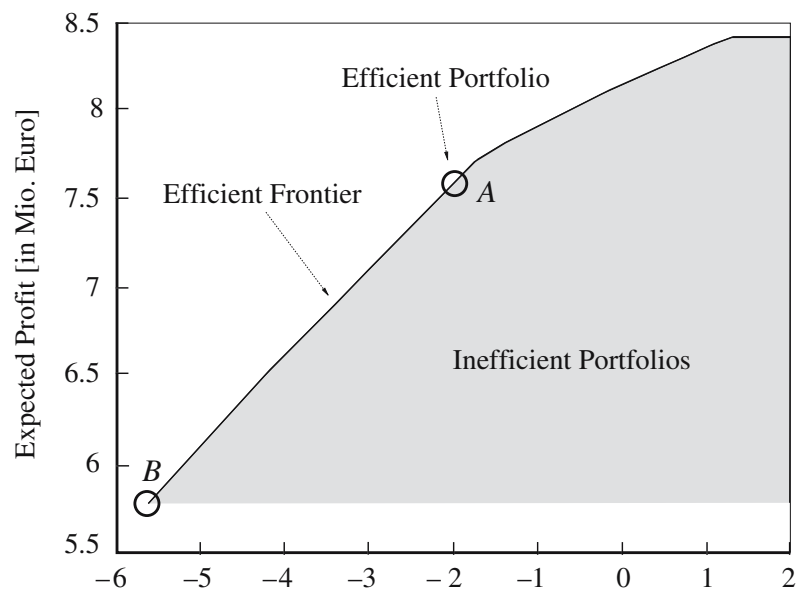

Fig. 8 Efficiency frontier of power portfolio optimization 

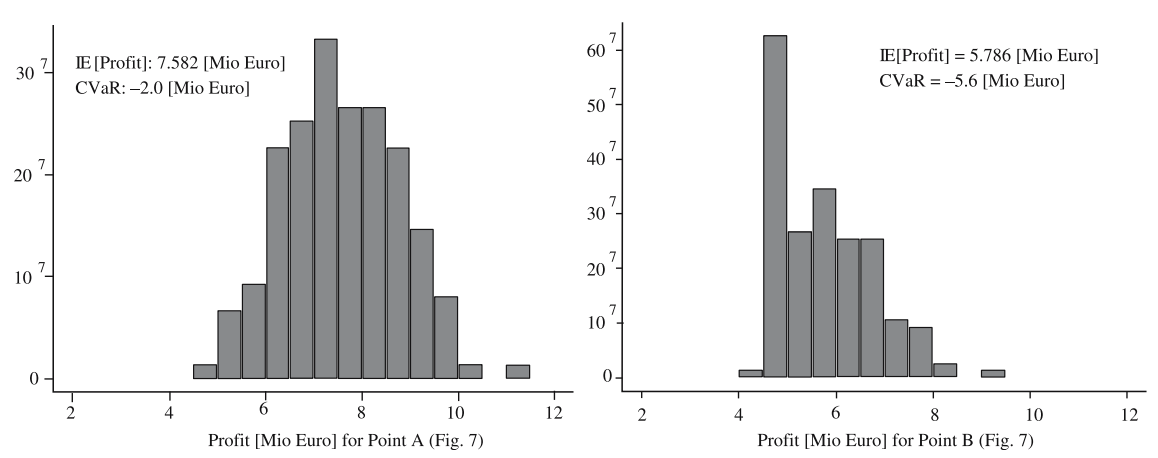

Fig. 9 Shift in P\&L distribution

What can we learn from the efficient frontier? By looking at the efficient frontier we observe the excellent hedging performance, i.e. risk reduction capability, of hydro power plants. Assume a strategic decision is made to decrease the level of risk that a company is willing to accept. This change in risk level $C$ is accompanied by a decrease in expected profit $\mathbb{E}[-l(\boldsymbol{x}, \boldsymbol{Y})]$ (from now on denoted as $E$ ) (see Fig. 8). Moreover a more risk averse strategy (point B in Fig. 8) leads to a right skewed and fat-tailed distribution (see Fig. 9 on the right). This impact on power portfolio optimization is impressive and the motivation for definition 1.

Denote $y_{C}^{*}$ the dual price associated with risk level $C$. Observe (under nondegeneracy) that $y_{C}^{*}$ is the slope of the efficiency frontier at risk level $C$ with the corresponding expected profit $E$. Using the well-established sensitivity framework of linear programming we can derive a direct relation between a change in risk $\Delta C$ and in expected profit $\Delta E$ (at a given risk level $C$ ).

$$
\Delta E=y_{C}^{*} \Delta C .
$$

Assuming $y_{C}^{*}$ to be unique and positive, re-arranging of the terms lead to the following equation

$$
\Delta C=\frac{1}{y_{C}^{*}} \Delta E .
$$

Hence, one unit of reduction in expected profit results in $\frac{1}{y_{C}^{*}}$ reduction of risk. We remark that in the framework of coherent risk measures, risk reduction can be directly achieved by adding an equivalent amount of risk free capital to the portfolio (see Artzner et al. 1999). This, for CVaR trivial, observation justifies the following definition:

Definition 1 The hedging value of flexibility, $\xi_{1}$, at risk level $C$ is defined as

$$
\xi_{1}=\frac{1}{y_{C}^{*}}
$$


One achieves a risk reduction of $\xi_{1}$ by reducing the expected profit of the portfolio by one unit. Looking at Fig. 8 , one observes that a change from position $\mathrm{A}$ to B leads to a hedging value of flexibility of $\xi_{1}=2$. Thus, one unit reduction of expected profit reduces the risk twice as much! Note, that as long as $\xi_{1} \geq 1$ (in a "risk-free" discounted model) it is certainly better to hedge against risk with flexibility instead of adding risk-free capital. Hence, one can say that the power producing company in the case study should use its flexibility to reduce the risk level $C$ from $C_{A}=-2.0$ million Euro to $C_{B}=-5.6$ million Euro instead of adding cash. A risk reduction can be obtained by making use of our methodology presented so far using e.g., a risk level $C=C_{A}$. This will lead to a policy which fulfills the new requirements. One could also interpret the results from the opposite side. Being risk-aggressive might not pay off. A company needs to quadruple its risk in order to double its expected profit.

\subsection{Marginal value of flexibility}

Until now we were only concerned about the relationship between expected profit $E$ and its corresponding risk level $C$. We gave a decision rule what should be done (i.e. use flexibility or risk-free capital) to hedge against risk. Flexibility of volume, which is certainly one of the most important aspects of production management, was neglected so far. Hence, we look at it by assessing its pure risk reduction capacity without changing expected profit $E$. That is, the expected profit $E$ is treated as constant, say as a management target.

Figure 10 is the motivation for the analysis to come: At a given volume $V_{1}$ that is used to generate electricity we achieve an expected profit $E_{1}$ at risk level $C_{1}$. If the volume is now increased the power producing company would earn an additional yield which would hence lead to a higher profit, i.e. $E_{2}$ with $E_{2}>E_{1}$. But as we require that the expected profit does not change this earning is used to reduce the risk from $C_{1}$ to $C_{2}$.

Towards that end a power producing company may ask the following question: Assumed that by an investment, such as the opening up of new inflow areas, we can

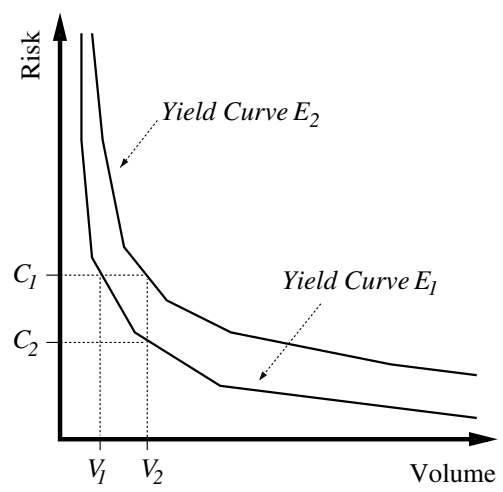

Fig. 10 Relationship between risk and volume 
use one additional unit of water in the power plant: What is this investment worth? We reflect the newly attained inflow by eliminating the sustainability constraint from our linear program and mirror this gain in additional inflow by lowering the end level of water $V_{\text {end }}$ by $\Delta V$ units. ${ }^{15}$ The additional unit of water can now be valued by the shadow price of the end water level constraint.

Again, using the sensitivity concept of linear optimization ${ }^{16}$ we can write for the change in expected profit:

$$
\Delta E=y_{C}^{*} \Delta C-y_{V_{\text {end }}}^{*} \Delta V
$$

where $y_{C}^{*}$ and $y_{V_{\text {end }}}^{*}$ are unique dual variables for the risk level $C$ and the end level of water constraint $V_{\text {end }}$ respectively. Assuming that the change in volume $\Delta V$ is only off-set by a reduction in risk $\Delta C$ as the expected profit $E$ is kept constant (i.e. $\Delta E=0)$ we obtain

$$
\Delta C=\frac{y_{V_{\text {end }}^{*}}^{*}}{y_{C}^{*}}=\Delta V=\xi_{2} \Delta V .
$$

Analog to the previous subsection we therefore define:

Definition 2 The marginal value of flexibility $\xi_{2}$ for one additional unit of volume is defined as

$$
\xi_{2}=\frac{y_{V_{\text {end }}^{*}}^{*}}{y_{C}^{*}}=\xi_{1} y_{V_{\text {end }}^{*}} .
$$

Within that framework we have obtained a leverage for the dual price of volume $y_{V_{\text {end }}}^{*}$ which we call marginal value of flexibility $\xi_{2}$ (c.f. Fig. 11). The tighter the risk constraint, the higher the marginal value of flexibility and the gain from an an additional volume unit. With $\xi_{1}=2$ we are able to double the dual price of volume $y_{V_{\text {end }}^{*}}^{*}$.

Reconsider the case of the power portfolio where $\mathrm{CVaR}^{17}$ was applied as a risk measure and let $\mathrm{P}^{E}$ denote the loss distribution of the optimal portfolio with respect to the expected profit $E$ at a given level of risk $C$. Changing the volume $V$ of the electricity production process (by e.g., decreasing the end level of water), making use of the translation invariance, and applying definition 2 leads to

$$
\rho\left(\mathrm{P}^{E}+\xi_{2} \Delta V\right)=\rho\left(\mathrm{P}^{E}\right)-\Delta C .
$$

This means that the additional volume $\Delta V$ is transformed by the marginal value of flexibility $\xi_{2}$ into an additional cash-flow which reduces the risk for "free." The cash-equivalence follows from the framework of coherent risk measures. Analog to the previous subsection this leads to the important observation: As long as

\footnotetext{
${ }^{15}$ Note that $\Delta V$ is always negative. A smaller end level of water leads to a higher volume as more water can now be used to produce electricity.

${ }^{16}$ Assuming once again non-degeneracy. For degenerated optimal points directional derivatives have to be applied. This additional step will not change the methodology we suggest.

${ }^{17}$ Any coherent or convex risk measure could have been used.
} 


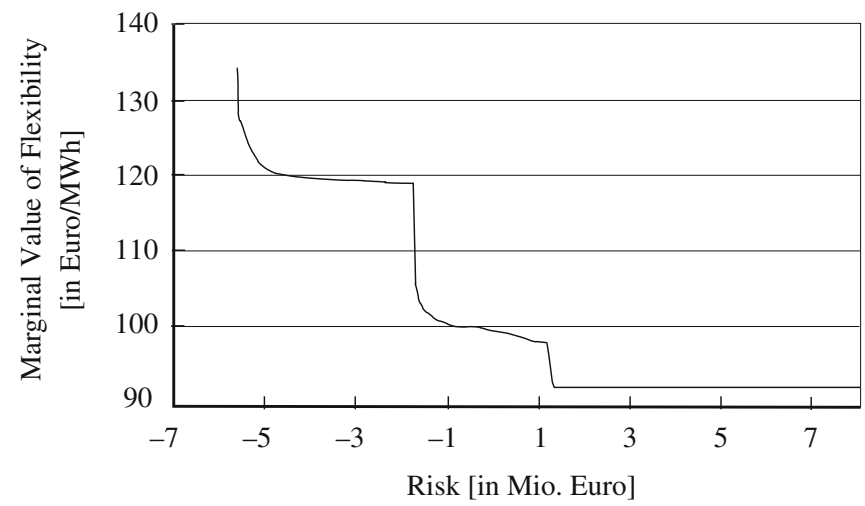

Fig. 11 Marginal value of flexibility

$\xi_{2} \geq 1$ (in a risk-free discounted framework) flexibility should be used to hedge against risk instead of adding risk-free capital.

\section{Conclusions}

In this paper we deduced a consistent way of thinking about risk and flexibility in the electricity market. Using the concept of coherent risk measures we presented a linear programming approach to optimize a power portfolio including financial contracts and physical assets. The result is a dynamic dispatch policy combined with an optimal contract portfolio. The advantage of our approach is the computational ease even for a large number of scenarios. Additionally our method allows us to make use of the well-known concept of duality in order to derive concepts for the valuation of flexibility.

In this setting, the value of flexibility is understood as the capacity to reduce risk, measured with $\mathrm{CVaR}$, and can hence be valued accordingly. At a direct level we observe the hedging value of flexibility by comparing a change in expected earnings with a change in acceptable risk, expressed by the inverse of the slope of the efficient frontier. At an indirect level, we looked at the marginal risk reduction capacity induced by operational components such as plant capacity keeping the earnings target fixed. We called this marginal flexibility because its value is derived by purely making use of local marginal concepts. Alternatively, one could have minimized the risk requiring a target earning so that the marginal value of flexibility would correspond to the dual price of the changing operational constraint. In any case, since risk reduction can be achieved either by adding risk-free capital to the portfolio or changing the operational level we set the marginal value of flexibility equal to the marginal risk-reduction capacity.

Based on those results we are currently working on expanding this model towards an integrated model encompassing various multi-stage hydro power plants, base load power plants, a supply area, and several spot markets. With this model any contract can be valued microeconomically from a utility point of view using seasonal dispatch policies and dynamic risk measures. 


\section{References}

Acerbi C, Tasche D (2002) On the coherence of expected shortfall. In: Szegö G (ed) Beyond VaR (Special Issue). Journal of Banking \& Finance 26:1505-1518

Arrow KJ (1971) Essays on the theory of risk-bearing. Markham, Chicago

Artzner P, Delbaen F, Eber J-M, Heath D (1999) Coherent risk measures. Math Financ 9:203-228

Bertsimas D, Lauprete GJ, Samarov A (2000) Shortfall as a risk measure: properties, optimization and applications. Working paper, MIT

Borenstein S, Bushnell J (1999) An empirical analysis of the potential for market power in California's electricity industry. J Ind Econ 47(3):285-323

Burger M, Klar B, Müller A, Schindlmayr G (2004) A spot market model for pricing derivatives in electricity markets. Quantitative Finance 4:109-122

Carmona R, Dayanik S (2004) Optimal multiple-stopping of linear diffusions and swing options. Preprint, Princeton University

Clewlow L, Strickland C (2001) Energy derivatives - pricing and risk management. Lacima Publications, London

Delbaen F (2000) Coherent risk measures. Lecture notes at Cattedra Galileiana. Scuola Normale di Pisa, Pisa

Deng S, Johnson B, Sogomonian A (2001) Exotic electricity options and the valuation of electricity generation and transmission assets. Decis Support Syst 30(3):383-392

Eberlein E, Stahl G (2003) Both sides of the fence: a statistical and regulatory view of electricity risk. Energy Power Risk Manag 8(6):34-38

Eydeland A, Geman H (2000) Fundamentals of electricity derivatives in energy modeling and the management of uncertainty. Risk Books, New York

Eydeland A, Wolyniec K (2002) Energy and power risk management. Wiley, New York

Fleten S-E, Wallace SW, Ziemba WT (2002) Hedging electricity portfolios via stochastic programming. Decision making under uncertainty: energy and power. Springer, Berlin Heidelberg New York, pp 71-94

Föllmer H, Schied A (2002) Convex measures of risk and trading constraints. Finance Stoch 6(4):429-447

Frey R, McNeil A (2002) VaR and expected shortfall in credit portfolios: conceptual and practical insights. In: Szegö G (ed) Beyond VaR (Special Issue). J Bank Financ 26:1317-1334

Geman H (2001) Spot and derivatives trading in deregulated European electricity markets. Rev Econ Soc 8

Gröwe-Kuska N, Römisch W (2002) Stochastic unit commitment in hydrothermal power production planning. Preprint 02-3, Institute for Mathematics, Humboldt-University Berlin

Güssow J (2001) Power systems operations and trading in competitive energy markets. PhD thesis, University of St. Gallen, Switzerland

Hinz J (2003) Optimizing a portfolio of power-producing plants. Bernoulli 9(4):659-669

Hinz J, von Grafenstein L, Verschuere M, Wilhelm M (2004) Pricing electricity risk by interest rate methods. Quant Financ (to appear)

Jaillet P, Ronn EI, Tompaidis S (2004) Valuation of commodity-based swing options. Manag Sci 50:909-921

Kamat R, Oren S (2002) Exotic options for interruptible electricity supply contracts. Oper Res 50(5):835-850

Kholodnyi VA (2004) Valuation and hedging of European contingent claims on power with spikes: a non-Markovian approach. J Eng Math 49:233-252

$\mathrm{Ku}$ A (2003) Risk and flexibility in electricity. Risk Books, London

Lüthi H-J, Doege J (2005) Convex risk measures for portfolio optimization and concepts of flexibility. Math Program, Series B 104(2-3):541-559

Markowitz HM (1952) Portfolio selection. J Finance 7:77-91

Mas-Colell A, Whinston M, Green J (1995) Microeconomic theory. Oxford University Press, New York

Pilipovic D (1997) Energy risk: valuing and managing energy derivatives. McGraw-Hill, New York

Rockafellar RT, Uryasev S (2000) Optimization of conditional value-at-risk. J Risk 2(3):21-41

Rockafellar RT, Uryasev S (2002) Conditional value-at-risk for general loss distributions. J Bank Finance 26:1443-1471

Rudin W (1976) Principles of mathematical analysis. McGraw-Hill, Singapore 
Schwartz ES, Lucia JJ (2002) Electricity prices and power derivatives. Evidence from the Nordic power exchange. Rev Deriv Res 5(1):5-50

Stoft S (2002) Power system economics. IEEE, Wiley-Interscience, New York

Thompson AC (1995) Valuation of path-dependent contingent claims with multiple exercise decisions over time: the case of take-or-pay. J Financ Quant Anal 30(2):271-293

Unger G (2002) Hedging strategy and electricity contract engineering, Ph D thesis, Swiss Federal Institute of Technology - ETH Zürich, http://www.ifor.math.ethz.ch/publications/ diss unger 